Research Paper

\title{
Physical and chemical characteristics of mucin secreted by pseudomyxoma peritonei (PMP)
}

\author{
Krishna Pillai ${ }^{\bowtie}$, Javed Akhter, Ahmed Mekkawy, Terence C Chua and David L Morris \\ Department of Surgery, University of New South Wales, St. George Hospital, Kogarah, Sydney, NSW, AUSTRALIA. \\ $\triangle$ Corresponding author: Krishna Pillai, Department of Surgery, University of New South Wales, St. George Hospital, Kogarah - NSW- Australia 2217. Tel \\ Number: 61 (02) 91132973 Email. panthera6444@yahoo.com.au. \\ (C) Ivyspring International Publisher. This is an open access article distributed under the terms of the Creative Commons Attribution (CC BY-NC) license \\ (https://creativecommons.org/licenses/by-nc/4.0/). See http://ivyspring.com/terms for full terms and conditions.
}

Received: 2016.06.08; Accepted: 2016.09.27; Published: 2017.01.01

\begin{abstract}
Background: Pseudomyxoma peritonei (PMP) is a rare disease with excess intraperitoneal mucin secretion. Treatment involves laparotomy, cytoreduction and chemotherapy that is very invasive with patients often acquiring numerous compromises. Hence a mucolytic comprising of bromelain and $\mathrm{N}$-acetyl cystein has been developed to solubilise mucin in situ for removal by catherization. Owing to differences in mucin appearance and hardness, dissolution varies. Therefore the current study investigates the inter-mucin physical and chemical characteristics, in order to reformulate an effective mucolytic for all mucin.

Method: PMP mucin, from the three categories (soft, semi hard and hard mucin) was solubilised and then various physical characteristics such as turbidity, density, kinematic viscosity were measured. The water content and the density of solid mucin were also determined. This was followed by the determination of sialic acid, glucose, lipid, Thiol (S-S and S-H) content of the samples. Lastly, the distribution of MUC2, MUC5B and MUC5AC was determined using western blot technique.

Results: Both turbidity and kinematic viscosity and sialic acid content increased linearly as the hardness of mucin increased. However, density, hydration, protein, glucose, lipid and sulfhydryl and disulphide content decreased linearly as hardness of mucin increased. The distribution ratio of mucins (MUC2:MUC5B:MUC5AC) in soft mucin is 2.25:1.5:1.0, semi hard mucin is $1: 1: 1$ and hard mucin is $3: 2: 1$.

Conclusion: The difference in texture and hardness of mucin may be due to cellular content, hydration, glucose, protein, lipids, thiol and MUC distribution. Soft mucin is solely made of glycoprotein whilst the others contained cellular materials.
\end{abstract}

Key words: pseudomyxoma peritonei, mucin, physical, chemical, hardness.

\section{Introduction}

Psedomyxoma peritonei (PMP) is a rare disease that occurs in 1 - 2 per million patients examined for peritoneal disease. The disease is commonly caused by tumour cells originating from appendix or less commonly from other sources such as colorectal cancers, ovarian or other cancer cells. [1, 2] Visual symptoms are usually the swelling of abdominal region caused by the accumulation of peritoneal mucinous ascites. If untreated, patients often succumb to nutritional insufficiency owing to compression set up by accumulating intraperitoneal mucin as well as blockage of digestive tract by mucin.[3, 4] Current treatment involves laparotomy, cytoreduction and intraperitoneal chemotherapy. [5] The five year survival of patients after treatment is in excess of $80 \%$ according to a recent study [6] and patients may often require subsequent treatment. Owing to the significant invasive surgical procedures, patients often end up with numerous compromises and morbidity. [2, 7-9] Hence, we have developed a 
mucolytic that can solubilise the mucin in situ and enable the removal of mucinous material through a less invasive process such as a peritoneal catheter.[10] Further, the mucolytic agent has potent cytotoxicity that may enable its use as a cytotoxic agent[11] thereby facilitating the removal of peritoneal mucin with cytotoxic treatment in a single process.

In our earlier investigation on 36 PMP patient mucin samples, we found that not all mucins were of similar texture, compactness, and hardness. The efficacy of our novel mucolytic also showed variability in the disintegration of the different samples, with majority showing complete disintegration whilst others showed a reduced mucolytic effect. Subsequently, we were able to classify the mucins based on visual inspection as well as on the compact nature of mucin, into three grades (Figure $1 \mathrm{~A}$ ). All soft mucin disintegrated into an amber coloured liquid after being treated with 300 $\mu \mathrm{g} / \mathrm{ml}$ bromelain and $250 \mathrm{mM} \mathrm{N}$-acetyl cystein (NAC) (Figure $1 \mathrm{~B}$ ), whilst the semi hard and the hard mucin disintegrated to 60 and $40 \%$, respectively. Hence, in the current work we aim to investigate the differences in physical and chemical composition of the three grades of mucin. This may enable us to further enhance the efficacy of the current mucolytic to solubilise all PMP mucin, regardless of their appearance or grades of hardness. To the best of our knowledge, this has never been carried out on PMP mucin before.

\section{Materials and Methods}

The experiment was conducted with approval from the St. George Hospital Ethics committee. Patient mucin samples were collected under sterile conditions and frozen immediately at $-80^{\circ} \mathrm{C}$, for storage. For experimental work frozen mucin was carefully thawed to room temperature in a warm water bath. For the purpose of this analytical work a total of 16 patient mucin samples were selected, 10 were soft, 3 were semi hard and 3 were hard mucin.

All chemical agents used in the current analytical work were procured from Sigma Aldrich Chemicals, Sydney, Australia. All antibodies were purchased from Santa Cruz Biotechnology Pty Ltd. CA, USA.

\section{Preparation of mucin for analysis}

From each mucin sample $1.0 \mathrm{~g}$ of sample was carefully weighed and inserted into a centrifuge tube containing $10 \mathrm{ml}$ TRIS buffer ( $\mathrm{pH}$ 7.0) The mucin was then shredded into small particles using an ultrasonic shredder (Ystral 0-879292, Ballrechten-Dottingen) for a brief $30 \mathrm{sec}$. Extraction of mucin and isolation of purified mucin were performed as described by Mall et al. [12] Briefly soluble mucin was treated with 10 $\mathrm{mmol} / \mathrm{L}$ dithiotreitol (DDT) in $6 \mathrm{~mol} / \mathrm{L}$ guanidinium hydrochloride $(\mathrm{GuHCl}), 5 \mathrm{mmol} / \mathrm{L}$ EDTA, $0.1 \mathrm{~mol} / \mathrm{L}$ Tris- $\mathrm{HCl}$ buffer, $\mathrm{pH} 8.0$ for 5 hours at $37^{\circ} \mathrm{C}$ for reduction of mucin and subsequently alkylated with $25 \mathrm{mmol} / \mathrm{L}$ idoacetamide (IAA) for 15 hours at room temperature in the dark.

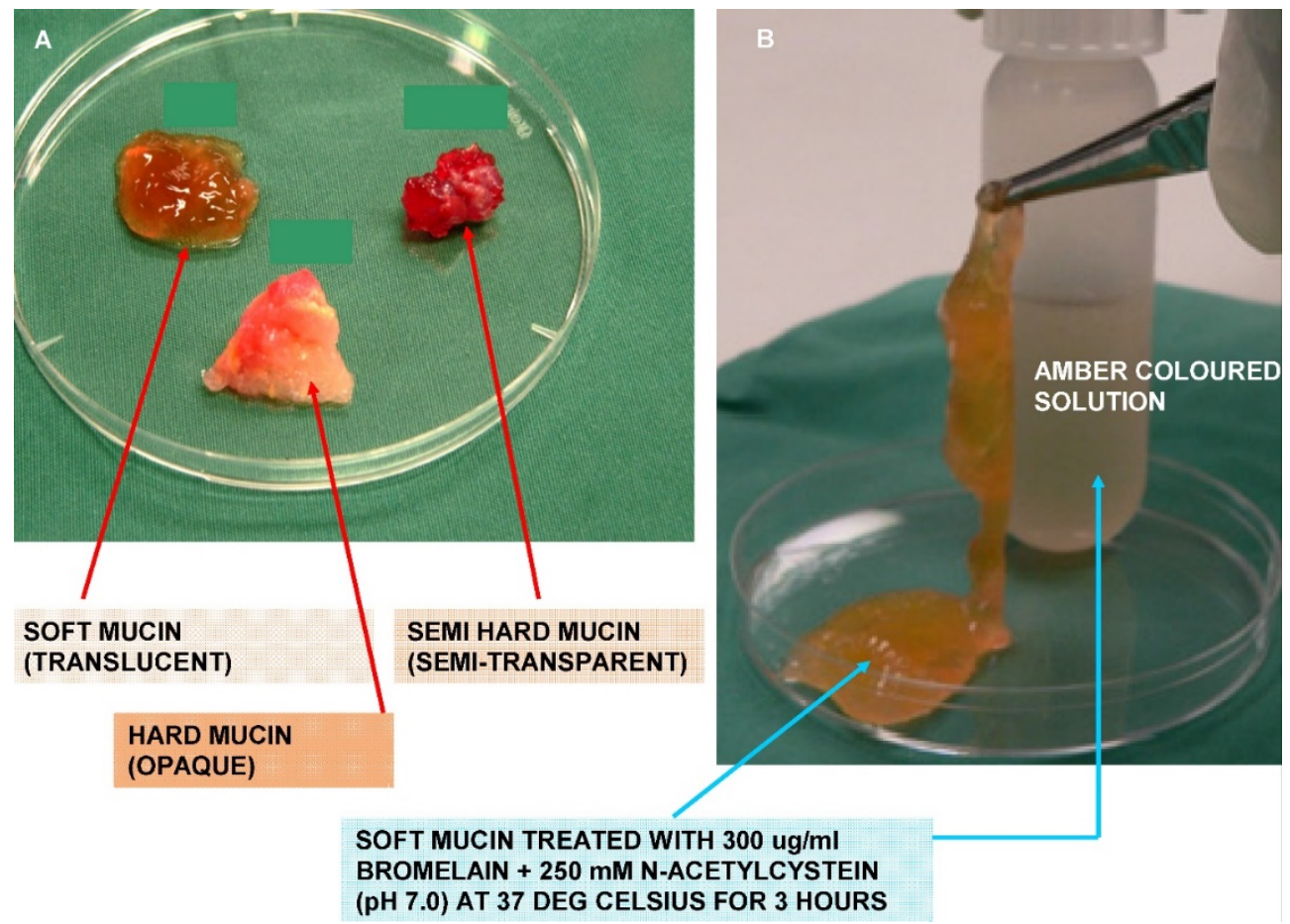

Figure 1. A show the appearance and texture of the three types (grades) of mucin. The soft mucin is almost transparent, semi hard is semitransparent whilst the hard being almost opaque. Figure $1 \mathrm{~B}$ show the transformation of soft mucin into an amber coloured liquid when treated with $300 \mathrm{ug} / \mathrm{ml}$ bromelain and $250 \mathrm{mM} \mathrm{N}$-acetyl cystein for 3 hours at $37 \mathrm{deg}$ Celsius. 
The solubilised reduced mucin was then subjected to density gradient in $3.5 \mathrm{~mol} / \mathrm{L}$ caesium chloride $(\mathrm{CsCl} / 4 \mathrm{~mol} / \mathrm{L} \mathrm{GuHCl})$ twice for 48 hours at $105,000 \mathrm{~g}$ with a starting density of $1.39-1.42$ $\mathrm{g} / \mathrm{ml}$.[13] The mucin rich fractions were pooled, dialysed against three changes of distilled water.

\section{Turbidity}

To measure the turbidity of solubilised mucin, a well mixed $500 \mu \mathrm{l}$ of each of the solubilised sample from the above preparation was transferred into a transparent cell (cuvette) and absorbance at 290nm was measured. The instrument was blanked with distilled water, prior to measuring absorbance of the mucin solutions. For each mucin grade, the mean turbidity with standard deviation was determined.

\section{Density (solubilised mucin)}

To a pre-weighed $2 \mathrm{ml}$ vial (ependorff) was added $1 \mathrm{ml}$ of the solubilised mucin solution. The difference in weight was recorded. Density was determined by dividing the weight recorded by the volume $(1 \mathrm{ml})$. For each mucin grade the mean density with standard deviation was determined.

\section{Kinematic viscosity}

The kinematic viscosity of each solubilised mucin sample was determined using methods by Fries et al.[14] Briefly the time in seconds and the height the liquid rises in an inclined capillary tube (1.0 $\mathrm{mm}$ in diameter) touching the surface of the liquid was measured at ambient room temperature of $21^{\circ} \mathrm{C}$. Mean values with standard deviation was determined for each grade of mucin.

\section{Percentage Water content (fresh mucin samples)}

A known weight $(1.0-1.5 \mathrm{~g})$ of each mucin sample was placed in a Petri dish and incubator dried (90 ${ }^{\circ} \mathrm{C}$ ) over 48 hours. The residual weight of the mucin was measured and percentage hydration was calculated using the below formula.

$$
\mathrm{N}=[(\mathrm{X}-\mathrm{Y}) / \mathrm{X}] 100
$$

Where: $\mathrm{N}=$ Percentage hydration; $\mathrm{X}=$ Weight of mucin before drying $(\mathrm{g}) ; \mathrm{Y}=$ Weight of mucin after drying $(\mathrm{g})$

Mean percentage of water for each grade of mucin was determined as before.

\section{Density (fresh mucin samples)}

The density of fresh samples were determined using the water displacement method, [15] Briefly 1.0 $\mathrm{g}$ of mucin sample was weighed and immersed in distilled water at room temperature, the density was determined using standard formula, Mass /Volume.
Mean value for each grade of mucin was determined, as before.

\section{Protein}

The protein content of mucin was determined using the method of Lowry et al. [16] Briefly, the mucin solution was prepared in a series of dilutions with distilled water $(1 / 50,1 / 100,1 / 500,1 / 1000)$ for protein determination. Standard curve was generated using bovine serum albumin at concentrations of 0,5 , $10,20,50,100 \mu \mathrm{g} / \mathrm{ml}$ ). Colorimetric measurements were carried out at $530 \mathrm{\eta m}$ with a spectrophotometer. The final concentration of protein was determined after adjustment to the dilution of the sample protein solution. Mean values for each grade of mucin were calculated.

\section{Sialic acid}

The sialic acid content of the solubilised mucin samples was determined using the BIO VISION Sialic Acid (NANA) Colorometeric/Fluorometeric Assay Kit (Milpatas, CA, USA). Solubilised mucin samples were diluted $(1 / 50,1 / 100,1 / 500,1 / 1000)$ and tested along with prepared sialic acid standard curve as recommended by the supplier. Absorbance (OD) was measured at 570 $\mathrm{nm}$. Mean values were generated for each grade of mucin using standard methods, as before.

\section{Glucose}

Glucose in the solubilised mucin samples are measured quantitatively using the phenol-sulphuric acid method as carried out by Masuko et al. [17] Briefly, $150 \mu \mathrm{l}$ of concentrated sulphuric acid and $30 \mu 1$ of $5 \%$ phenol in water were added in rapid succession to $50 \mu \mathrm{l}$ of mucin solution (dilution: 1/50, $1 / 100,1 / 200,1 / 500)$ in a microwell plate. After incubation for 5 minutes at $90 \circ \mathrm{C}$ in static water bath by floating the microplate carefully. The plate was then cooled to room temperature for $5 \mathrm{~min}$ in a water bath and wiped dry to measure absorbance at $4937 \mathrm{~m}$ by a microplate reader. Standard curves were prepared for glucose $(0,20,40,60,80,100 \mu \mathrm{g} / \mathrm{ml})$ in a similar fashion. Mean values were generated, using methods as before.

\section{Lipid}

The mean lipid concentration in the solubilised mucin samples were determined using the Cholesterol Quantification kit (SIGMA ALDRICH catalogue No. MAK403) in a 96 micro well plate. Briefly, using cholesterol standards, a standard curve was generated for the detection of $0,0.1,0.2,0.3,0.4$ and $0.5 \mathrm{ng} /$ well and solubilised mucin samples were diluted to read within this level. Colorimetric readings, Absorbance (OD) were carried out at 570 
$\eta \mathrm{m}$ following protocol as recommended by the manufacturer. Mean values were calculated for each mucin grade, as before.

\section{Thiol (S-S and S-H)}

To measure total sulfhydryl (SH) content, $0.5 \mathrm{~g}$ of mucin was carefully weighed into a $50 \mathrm{ml}$ centrifuge tube and reduced with $0.33 \mathrm{M} \mathrm{NaBH} 4$ in $8 \mathrm{M}$ Urea/20mM Na 2 EDTA/0.1 M NaH${ }_{2} \mathrm{PO}_{4} / \mathrm{Na}_{2} \mathrm{HPO}_{4}$, $\mathrm{pH} 9.0$ in a final volume of $5 \mathrm{ml}$. The mucin sample was initially broken up by sonification in the reducing media and incubated with gentle agitation in a water bath at $37 \circ \mathrm{C}$ for 90 minutes. A few drops of octan-1-ol were added to prevent foaming. After adding $1.5 \mathrm{ml}$ of $20 \%$ (w/v) SDS, excess $\mathrm{NaBH} 4$ was destroyed by titrating with acetic acid to pH.5.4 with further incubation of the mixture at 37 o $\mathrm{C}$ for 15 minutes. This was followed by addition of $1.5 \mathrm{ml}$ of 4,4'- dipyridyl disulphide (PDS) (Sigma chemicals, Australia) prepared in $0.2 \mathrm{mM}$-Sodium acetate solution pH. 5.0 and after further incubation at room temperature $(21 \circ \mathrm{C})$ for 30 minutes, the absorbance (OD) of the solution was measured at $324 \mathrm{\eta m}$ against a blank containing all reagents except mucin. Calculation of total $\mathrm{SH}$ content of mucin was determined by using molar absorption coefficient of $19800 \mathrm{M}^{-1} \mathrm{~cm}^{-1}$

For determining free $\mathrm{SH}$ groups (without including the reduced S-S groups), $0.5 \mathrm{~g}$ of mucin was carefully weighed in a $50 \mathrm{ml}$ centrifuge tube. Samples were dissolved (sonfication) in $5.0 \mathrm{ml}$ of $20 \mathrm{mM} \mathrm{Na} 2$ EDTA/2\% (w/v) SDS/0.2 M-Sodium acetate, pH.5.4 and incubated at $37 \circ \mathrm{C}$ for 15 minutes. On addition of

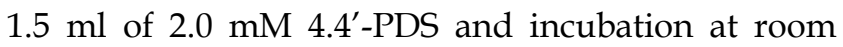
temperature for 30 minutes, the absorbance was measured at $324 \mathrm{~nm}$ against reagent blank, as before. Free S-H content was determined as before. The S-S bond content of mucin ( $\eta \mathrm{mol})$ was determined by subtracting the total amount of $\mathrm{SH}$ minus the free $\mathrm{SH}$.

\section{Detection of MUC2, MUC5B and MUC5AC by Western blot}

Equal quantity of solubilised mucin (based on protein concentration) were loaded on a gel and protein components were separated electrophoretically using standard methods and resolved on to a membrane. The membrane was blocked with $10 \%$ skim milk after which the separated components were probed with the respective primary antibodies to MUC2 (H-300, Sc.8827, goat polyclonal IgG), MUC5B (513\# 19-25, mouse monoclonal IgG) and MUC5AC (45M1, sc 21701, mouse monoclonal $\operatorname{IgG}$ ), as recommended by the manufacturer (Santa Cruz Biotech. Pty Ltd,). The secondary antibodies used were rabbit polyclonal that was supplied by Gen
Search Pty Ltd.

\section{Results}

All data have been plotted against hardness index (HI) a measurement system that has been developed by our group to classify the hardness of PMP mucin into three categories according to the area, a unit weight $(\mathrm{g})$ of mucin fully hydrated (soaked for 30 mins in distilled water) occupies when placed on a gridded glass slab (marked in $\mathrm{mm}$ ). The HI index of $<0.6=$ soft mucin; $>0.6-1.2=$ semi hard mucin and $>1.2=$ hard mucin (publication in press)

\section{Turbidity}

The turbidity measurements indicate that of the three grades of mucin solutions, soft mucin has the least turbidity whilst hard mucin was most turbid (Table 1, Figure 2 A). The turbidity plot with hardness index of each mucin grade indicates a linear relationship. Turbidity indicates the proportion of solids or components that deflect or absorb light and hence, the hard mucin seems to contain the maximum light absorbing components.

\section{Density}

Density measurements were carried out for both the solubilised mucin as well as for the solid mass of mucin. Density of the three grades of mucin as determined on the solid mass indicate that there was at least a $21 \%$ difference between the soft mucin and hard mucin $[(1.029-0.809) / 1.027 \times 100]=21 \%$. (Table 1) The densities of solubilised mucin indicate only a smaller difference $(9.8 \%)$ between the soft and hard mucin. The difference between the densities of solid mucin and mucin solution may indicate that materials may be trapped in the solid mucin and that is removed during solubilisation of the mucin. On the other hand a solid mucin with compacted proteins owing to intermolecular linkages may actually show a much reduced volume in relation to its weight and hence giving a much larger density value (Fig. 2 B \& C, Table 1).

\section{Kinematic viscosity}

Measurements of the kinematic viscosity (KV) of the solubilised mucin indicate that there is at least a $29 \%$ difference in the viscosities between soft and hard mucin. This indicates that solubilised hard mucin may be more viscous compared to soft mucin, further indicating that other components found within may be contributing to this viscosity. The hard mucin may contain viscous components that may give rise to this large difference between the soft and hard mucin solution (Table 1, Fig 2 D). 
A tURBidity VARIATION IN THE tHREE GRADES OF MUCIN

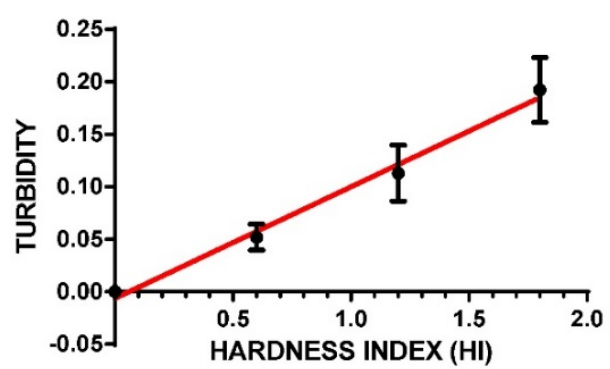

\begin{tabular}{|l|l|}
\hline Slope & $0.1063 \pm 0.007300$ \\
\hline Y-intercept when $\mathrm{X}=0.0$ & $-0.006404 \pm 0.008194$ \\
\hline $\mathrm{X}$-intercept when $\mathrm{Y}=0.0$ & 0.06022 \\
\hline 1/slope & 9.404 \\
\hline
\end{tabular}

B DENSITY VARIATION IN THE THREE GRADES OF SOLUBILISED MUCIN

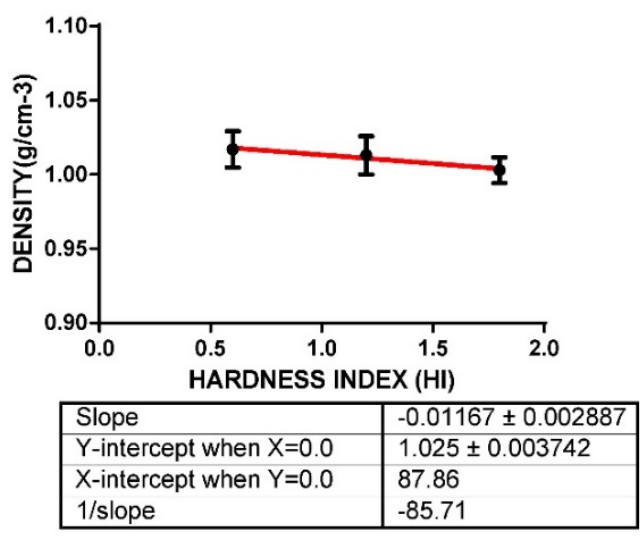

\section{DENSITY VARIATION IN THE THREE GRADES OF NON SOLUBILISED MUCIN}

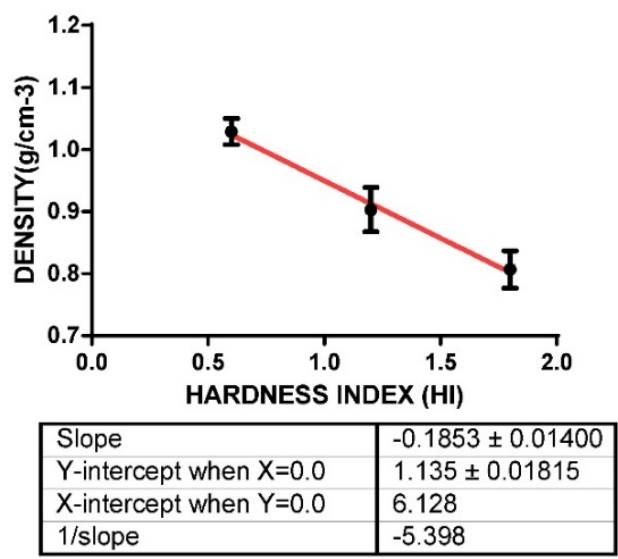

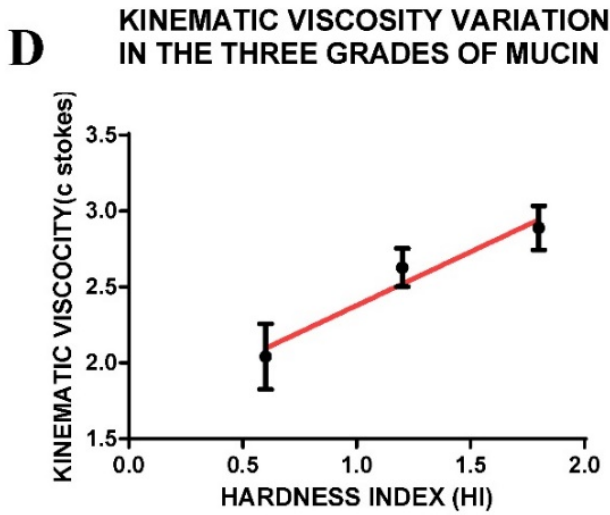

\begin{tabular}{|l|l|}
\hline Slope & $0.7058 \pm 0.1564$ \\
\hline Y-intercept when $\mathrm{X}=0.0$ & $1.673 \pm 0.2027$ \\
\hline $\mathrm{X}$-intercept when $\mathrm{Y}=0.0$ & -2.370 \\
1/slope & 1.417 \\
\hline
\end{tabular}

E PERCENTAGE OF HYDRATION VARIATION IN THE THREE GRADES OFMUCIN

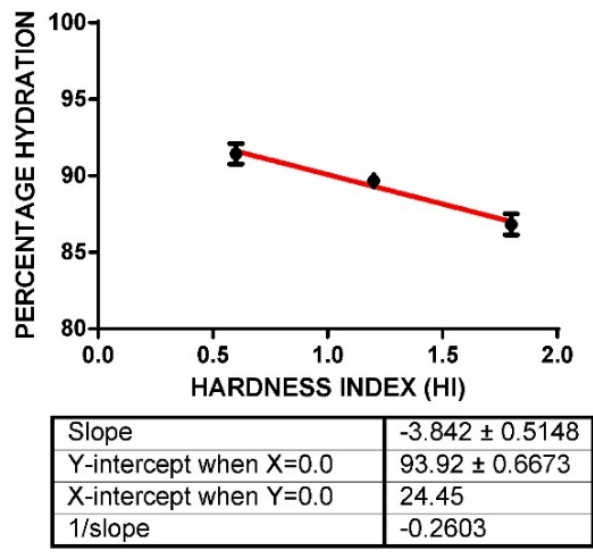

F PROTEIN CONTENT VARIATION IN THE THREE GRADES OF MUCIN

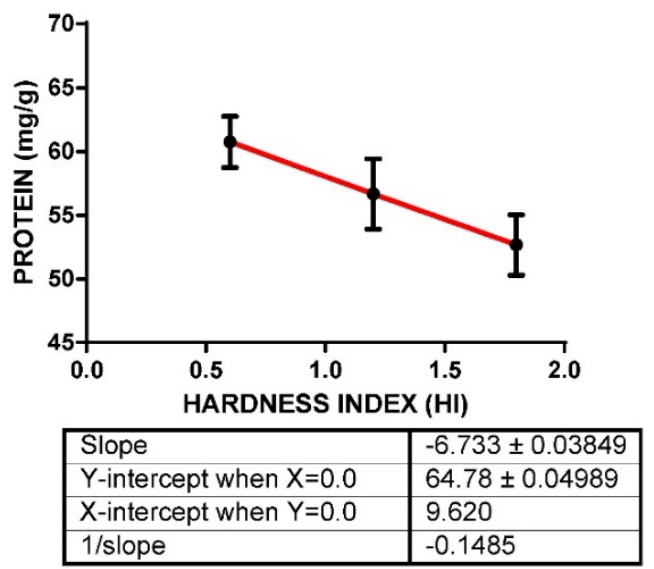

Figure 2. A shows turbidity variation within the three grades of mucin as represented by hardness index; $B$ that for density of solubilised mucin; $C$ that for density of solid mucin; D that for kinematic viscosity; $\mathrm{E}$ for hydration; $\mathrm{F}$ for protein content. The hardness index $(\mathrm{HI}) 0.6=$ soft mucin, $(\mathrm{HI}) 1.2=$ semi hard mucin and $(\mathrm{HI})$ $=1.8=$ hard mucin. 
Table 1. Mean value with SD for the various parameters measured.

\begin{tabular}{|c|c|c|c|c|c|}
\hline PARAMETER & SOFT MUCIN $(X)$ & SEMI HARD MUCIN (Y) & HARD MUCIN (Z) & $\mathrm{A}(\%)$ & $X: Y: Z$ \\
\hline Turbidity (OD 290nm) & $0.0518 \pm 0.01236$ & $0.1150 \pm 0.0266$ & $0.1923 \pm 0.0309$ & 73 & $1: 2.2: 3.7$ \\
\hline Density(sol)g/cm-3 & $1.017 \pm 0.0122$ & $1.013 \pm 0.0129$ & $1.003 \pm 0.0088$ & 13 & 1.01: $1.01: 1$ \\
\hline Density (solid)g/cm-3 & $1.029 \pm 0.029$ & $0.903 \pm 0.0357$ & $0.807 \pm 0.0376$ & 21.6 & $1.27: 1.12: 1$ \\
\hline Kinematic viscosity (cs) & $2.042 \pm 0.0216$ & $2.628 \pm 0.0126$ & $2.889 \pm 0.0440$ & 29.3 & 1: $1.29: 1.41$ \\
\hline$\%$ Hydration & $91.44 \pm 0.6603$ & $89.67 \pm 0.3844$ & $86.83 \pm 0.6820$ & 5 & 1.05: $1.03: 1$ \\
\hline Protein (mg/g) & $60.75 \pm 2.018$ & $56.67 \pm 3.360$ & $52.67 \pm 2.364$ & 13 & 1.15: $1.11: 1$ \\
\hline Sialic acid $\mu \mathrm{mol} / \mathrm{g}$ & $3.276 \pm 0.3856$ & $7.727 \pm 0.4175$ & $10.88 \pm 0.4156$ & 70 & $1: 2.36: 3.32$ \\
\hline Glucose (ng/g) & $9.1 \pm 0.1258$ & $7.8 \pm 0.1258$ & $6.167 \pm 0.2552$ & 32 & $1.45: 1.25: 1$ \\
\hline Lipid $(\mu g / g)$ & $525 \pm 40.54$ & $406.7 \pm 52.84$ & $300 \pm 28.74$ & 42.8 & 1.75: $1.35: 1$ \\
\hline Sulfhydryl ( $\mu \mathrm{mol} / \mathrm{g})$ & $310 \pm 22.8$ & $196.6 \pm 14.6$ & $129.4 \pm 12.9$ & 58.2 & 2.40: $1.52: 1$ \\
\hline Disulphide $(\mu \mathrm{mol} / \mathrm{g})$ & $280.6 \pm 21.9$ & $169 \pm 19.8$ & $114.3 \pm 17.8$ & 59.3 & 2.45: $1.47: 1$ \\
\hline
\end{tabular}

low value / high value) 100; CS = centistokes. Measurements of various parameters were carried out as outlined in the methods section.

\section{Hydration}

Measurement of percentage hydration (water present) between the three grades of mucin indicates that there is very little difference between them, only a $5 \%$ difference between the soft and hard mucin. The percentage hydration may depend on glycoprotein content that is capable of imbibing water, whilst cellular components may also be hydrated. Hence, although soft mucin generally appears to be jelly like and hence capable of imbibing water, the hard and semi hard mucin with its high cellular content may also have the potential to retain water. Hence, in total, there is only a slight difference between the soft and hard mucin (Table 1, Fig 2E).

\section{Protein}

Of the three samples analysed, the soft mucin contained the highest concentration of protein $(60.75 \mathrm{mg} / \mathrm{g})$ with hard mucin containing the least $(52.67 \mathrm{mg} / \mathrm{g})$ a difference of $13 \%$. There seems to be linearity in relationship between hardness index of mucin grades and protein concentration. This result may reflect on the proportion of mucin present in the three grades of mucin, soft mucin being composed of mainly glycoprotein as compared to the others that have cellular debris and other materials incorporated into the mucin mass and hence, the protein content variation between the grades of mucin is not very large (Fig 2 F, Table 1).

\section{Sialic acid}

There is a very large difference between the three grades of mucin in their sialic acid content, the semi hard mucin containing about twice that of soft mucin whilst the hard mucin contains three times that of soft mucin (Fig. 3 A, Table 1). Sialic acid or Nacetylneuramic acid may be related to the pathological state of the mucin.

\section{Glucose}

Glucose measurements indicate a linear decrease in concentration with increase in hardness index of the mucin sample (Fig. 3 B, Table 1); indicating that soft mucin contains almost a $30 \%$ higher amount of glucose compared hard mucin. This may be due to the higher content of mucinous material (glycoprotein) compared to semi hard and hard mucin that contains about $40-60 \%$ of cellular materials.

\section{Lipid}

The lipid content of the three grades of mucin seems to fall as the hardness index increases such that the soft mucin contains almost about $40 \%$ more lipids compared to hard mucin (Fig $3 \mathrm{C}$, Table 1). The presence of high level of lipids may have some bearing on the texture of mucin.

\section{Thiol (S-S and S-H)}

The total thiol content (sulfhydryl + disulphide) content of the mucin suggests that soft mucin has a much higher level compared to either the semi hard or hard mucin. The sulfhydryl (free S-H) indicates that there is a $58.2 \%$ difference between the soft and the hard mucin, whilst the disulphide content also indicates that soft mucin has a higher level compared to the rest, the difference between soft and hard mucin being $59.3 \%$. The ratios of $\mathrm{S}-\mathrm{H}$ bonds in the three mucin grades, soft: semi hard: hard is 2.4: 1.52:1, similarly the ratios for the S-S bond concentration are 2.45: 1.47: 1 , indicating that a similar ratio S-H: S-S exists in the three grades of mucin (Fig. 3 D, Table 1). However, there is a slightly lower level of S-S groups compared to $\mathrm{S}-\mathrm{H}$, in the three grades of mucin, difference being $9.3 \%$ for soft, $14.2 \%$ for semi hard and $11.6 \%$ for hard mucin, indicating that a greater number of S-S relative to S-H groups are found in soft mucin. 


\section{Western blot analysis}

All three MCU2, 5B and 5AC were found in the three grades of mucin (Table 2 and Figure 4 A), however the relative presence of these mucins in the patient samples were different in the three grades of mucin. The relative presence of these mucins is shown in Figure 4 B, C \& D, soft mucin having 90\% MUC2, $60 \%$ MUC5B and 40\% MUC5AC. In the semi hard mucin, all the three MUCs are present equally in the patient samples. Finally, in the hard mucin MUC2 (100\%), MUCB (33.3\%) and MUC5AC (66.6\%) is present.

The relative presence of MUCs may have an implication on the pathological state of the different grades of mucin, as well as the hardness of mucin.

\section{A glucose VARIATION IN THE three GRADES OF MUCIN}

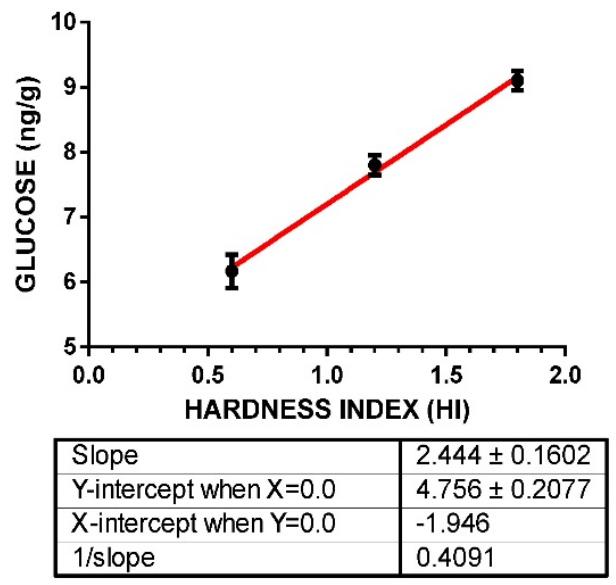

\section{B SIALIC ACID VARIATION IN THE THREE GRADES OF MUCIN}

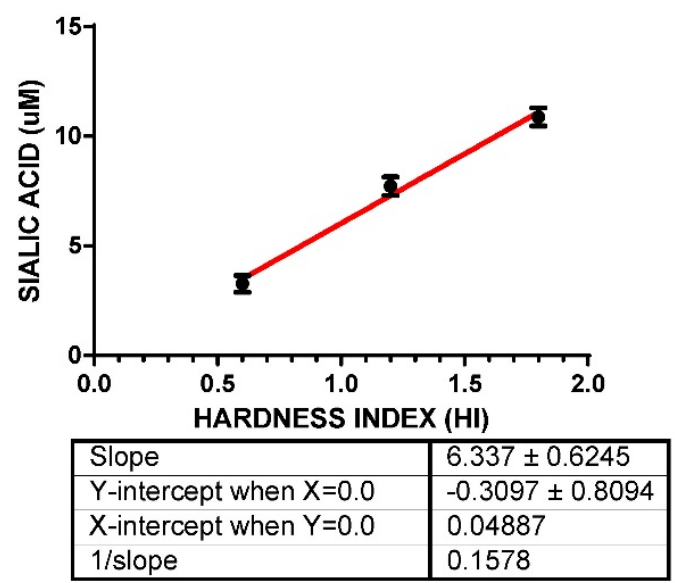

Table 2. Distribution of MUC2, MUC5B, MUC5AC in the different grades of pseudomyxoma peritonei mucin samples.

\begin{tabular}{lllll}
\hline Patient No & MUCIN GRADE & MUC2 & MUC5B & MUC5AC \\
\hline 1 & soft & - & - & - \\
2 & Soft & + & + & + \\
3 & Soft & + & - & + \\
4 & Soft & + & + & + \\
5 & Soft & + & + & - \\
6 & ooft & + & + & - \\
7 & Soft & + & + & - \\
8 & Soft & + & + & + \\
9 & soft & - & - & - \\
10 & Soft & + & + & - \\
11 & Semi hard & + & + & + \\
12 & Semi hard & + & + & + \\
13 & Semi hard & + & + & + \\
14 & Hard & + & - & + \\
15 & Hard & + & + & - \\
16 & hard & + & - & + \\
\hline$(+)=$ presence & or (-) absence of specific protein. &
\end{tabular}

\section{LIPID VARIATION IN THE THREE GRADES OF MUCIN}

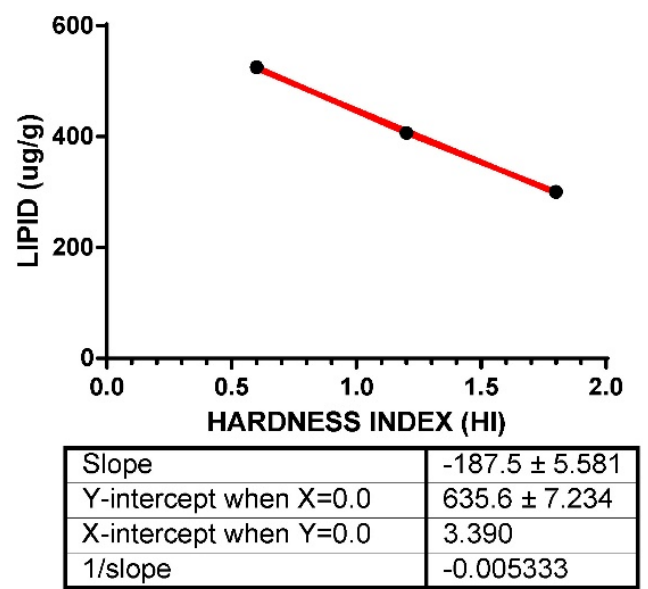

\section{SULPHYDRYL (SH) AND DISULPHIDE (SS) VARIATION IN THE THREE GRADES OF MUCIN}

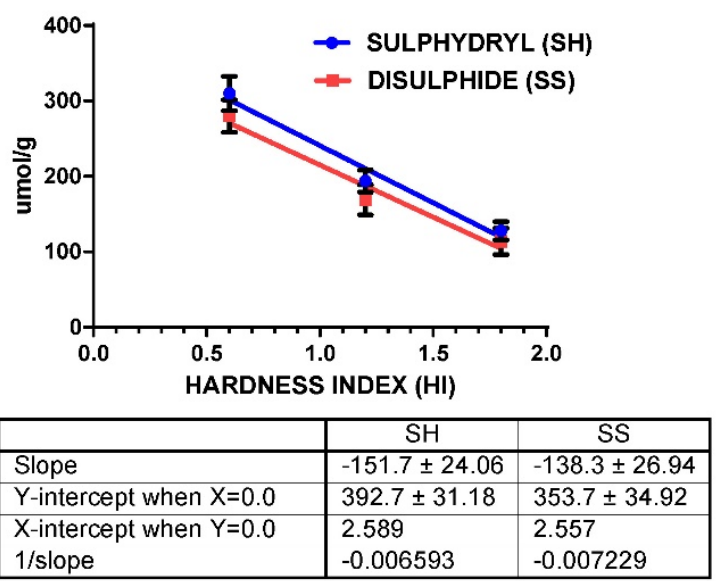

Figure 3. A shows the mean glucose content for the three grades of mucin; $B$ for sialic acid content; $C$ for lipid content and $D$ for thiol content (S-H and S-S). The hardness index $(\mathrm{HI}) 0.6=$ soft mucin, $(\mathrm{HI}) 1.2=$ semi hard mucin and $(\mathrm{HI})=1.8=$ hard mucin 
A
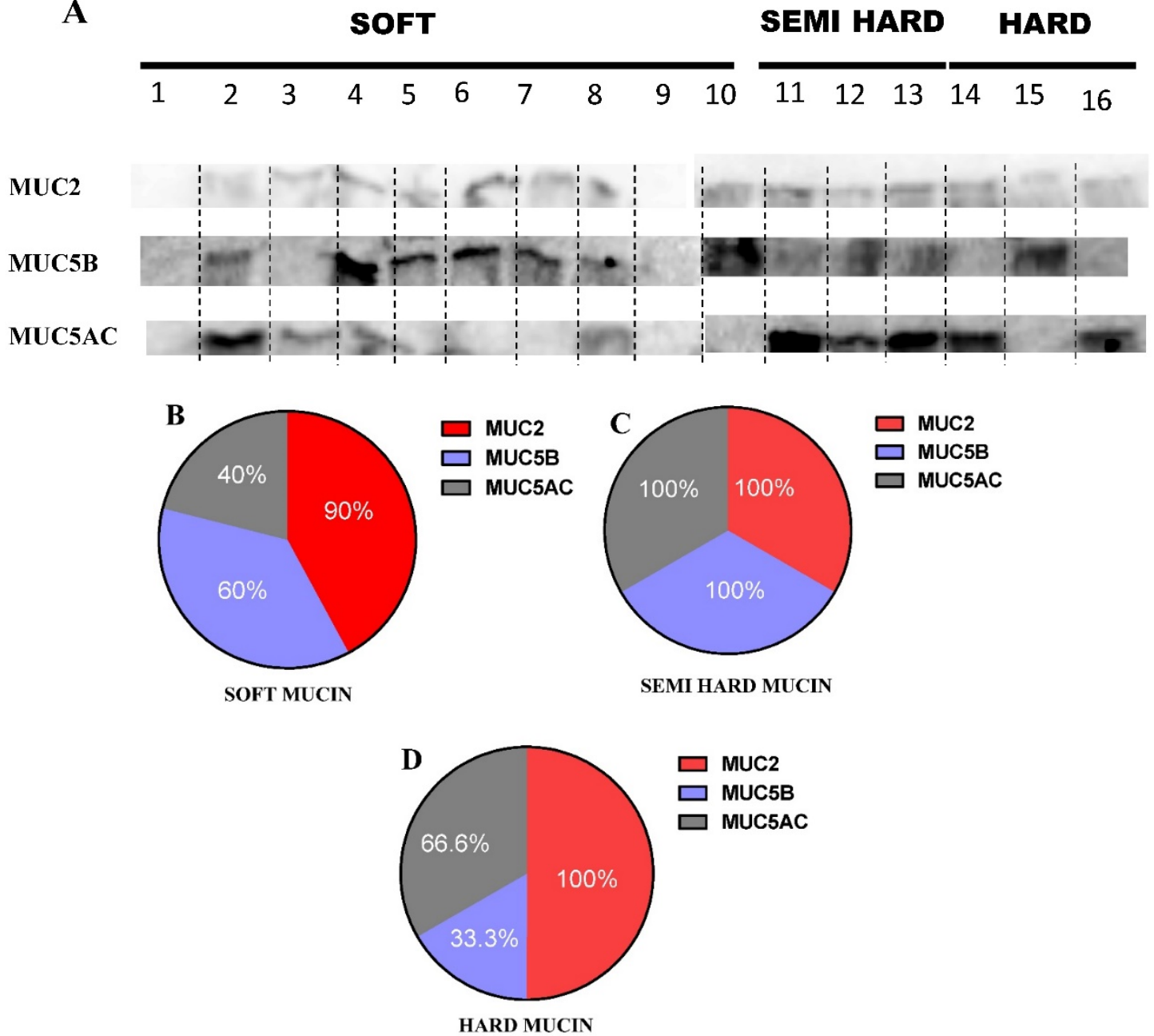

Figure 4. A shows the presence or absence of MUC2,MUC5B and MUC5AC in the three grades of mucin that was analysed in 16 patient mucin samples using western blot analysis. B, C \& D shows the percentage expression of the three types of mucin MUC2, MUC5B and MUC5AC in the three grades of mucin.

\section{Discussion}

We developed a mucolytic comprising of 300 $\mu \mathrm{g} / \mathrm{ml}$ bromelain and $250 \mathrm{mM}$-acetylcystein for in situ lysis of PMP mucin with evaluation in both, in vitro and in vivo studies. [10] The majority (62\%) of mucin were soft in texture and amenable to a $100 \%$ disintegration allowing possible removal through peritoneal catheters. The remaining samples disintegrated to about $40-60 \%$, with residual material left behind that appeared to be of cellular in nature. In an earlier study, the mucin was classified into three categories based on their physical appearance and their score in hardness index that was specifically developed in our laboratory to categorize mucin. (Paper in press) Hence, the present study was conducted to determine how the physical and chemical characteristics varied within the three grades of mucin samples in order to enable reformulation for dissolution of all mucin types, regardless of their appearance or hardness.

The results indicate that the three grades of mucin vary in several parameters that may influence their dissolution. The turbidity of solubilised mucin between the three grades of mucin showed that as the mucin became more compact or hard (as indicated by the hardness index), the turbidity of the solubilised mucin seems to increase, the difference between soft mucin and hard mucin being about $73 \%$. Turbidity is generally contributed by materials that are opaque or semi translucent while others that reflect light may also contribute to this common phenomena in solution.[18] Hence, this measure indicates that solid mucin contains a higher percentage of opaque components compared to the soft mucin. Although, the solubilised mucin was centrifuged to remove cellular fragments and other solids, there may be other materials that were capable of deflecting or absorbing light in the mucin.

Measurements of density of solubilised mucin indicated that that there was a minor difference between the three grades of mucin, the difference between soft and hard being about $13 \%$, suggesting a 
small variable composition between the grades that may contribute to this difference. However, the difference between the three mucins evaluated for density, in the solid form, seems to be more pronounced, difference between soft and hard being about $21 \%$, with soft mucin being much denser. The higher percentage of mucinous material with potential for greater hydration in soft mucin compared to the other grades of mucin, may contribute to the difference, However, the difference in hydration between the soft and semi hard mucin is very small (approximately 1\%) whilst that between the soft and hard mucin is around 5\%. Hence hydration may not alone contribute to the difference in densities. On the other hand, the semi hard and hard mucin may carry components that are less dense such as incorporation of air bubbles or even cellular components that may be slightly dehydrated (dead cells).

The kinematic viscosity suggested that hard mucin once solubilised may be more viscous compared to soft mucin. This may be due to the higher concentration of sialic acid present in the hard mucin. 16,17 There was a $70 \%$ difference between soft mucin and hard mucin in sialic acid content. The lipid content in soft mucin was much higher compared to hard mucin (42\% difference) and whether this lipid level reduced the kinematic viscosity of solutions needs investigation. The high viscosity may also be due to remanent cellular debris found within the hard mucin or other unidentified components found within.

The protein, glucose and lipid content of the three grades of mucin varied linearly with hardness index suggesting that they may have some bearing on the texture and hardness of the mucin. A higher protein content suggested a higher percentage of glycoprotein in the mucin sample and this meant that it may also attract a higher percentage of water, [19]. Hydration although may provide a higher mass to the tissues, it may also soften the mass of mucin through its hydrolytic forces that may disrupt inter molecular bonding linkages between the glycoprotein molecules.[20] The presence of lipid in the mucin may further reflect on the hardness since lipids may interfere with the formation of cross linkages between the protein molecules, [21] thereby reducing the compactness of the mucin sample. Hence, the high lipid content of soft mucin may in fact be softening the mucin texture and compactness. Sialic acid has been measured to be rather high in the hard mucin compared to the soft; its exact implication in the texture of the mucin needs to be determined in future studies. In mammalian glycoprotein, sialic acid occurs at the terminal end of the oligosaccharide side chains of the glyco -conjugates and imparts an electronegative charge to the mucin molecule. Owing to the weakness of its glycosidic linkage to the carbohydrate side chains, sialic acids are readily cleaved from such side chains with mild hydrolysis. Thus the concentration of free sialic acid may be used as a measure of desialyation of the conjugates [22] and hence degradation.[23] On the other hand, high sialyation with a larger concentration of negative charges may have some bearings on the compact structure that is seen in both semi hard and hard mucin.

The thiol content of the mucin grades suggests that soft mucin has a much higher percentage of both S-H and S-S concentration compared to the semi hard or the hard mucin. The difference between the soft and the hard mucin in the thiol concentration was almost $60 \%$ with soft mucin having the higher percentage. This indicates that the thiol concentration may not have a bearing on the mucin texture or hardness. Thiols generally contribute to the disulphide linkages between the mucin chain and hence form the gelatinous mass [24,25]. In the present case, a much higher cellular content is found in both the semi hard and hard mucin compared to complete absence of cellular material in the soft mucin. This also implies that there is a much higher percentage of mucin in the soft variety (on a gram basis) compared to the other two forms. Hence, this may explain why we found a higher concentration of thiols in the soft mucin Further, there was also an indication that the ratio of S-H to S-S bonds in the three grades of mucin was equal, i.e. a 1:1 implying that the ratio of these two types of bonds may not have a bearing on the variation in texture and hardness seen in these mucin samples, implying that cellular content found within may be a major contributor.

The distribution of three MUCs (MUC2, MUC5B and MUC5AC) suggests that they are differentially distributed within the three grades of mucin. This may have some implication on the texture of the presenting mucin, although with a greater implication on the pathological state of the mucin. Examining the ratio of distribution of the three MUCs within the three grades of mucin, soft mucin has MUC2 : MUC5B: MUC5AC in the ratio of $2.25: 1.5: 1.0$; for semi hard mucin the ratio is $1: 1: 1$ and finally for hard mucin it is $3: 2: 1$. Whether these differential ratios of MUCs presence within the mucin contribute to variation in texture and hardness is a question to be answered in future studies. (Figure $3 \mathrm{~A}, \mathrm{~B}, \mathrm{C}, \mathrm{D})$. Mall et al. has reported the presence of MUC 4 [26], in his studies with a PMP patient. MUC 4 has been reported to have prognostic significance in pancreatic cancer $[27,28]$ and it may hold a similar role in PMP. 
Although MUC 4 is a transmembrane mucin, it may have some bearing on the texture of mucin displayed. Our future studies will incorporate the identification of this mucin and its role in PMP.

Hence, examining the present analysis, there was a suggestion that the major components that may contribute to mucin texture and hardness difference may lie in the differential distribution of sialic acid, glucose, proteins and lipids, whilst other components of cellular origin may also be a contributing factor. It appears that the soft mucin may have a higher percentage of mucinous material in the form of glycoprotein since analysis has indicated a higher content of protein and glucose compared to the other types of mucin. Further there was no cellular debris found in the soft mucin when compared to semi hard and hard mucin that carried varying quantities of cellular debris. Hence, the present analysis suggests that soft mucin was completely solubilised because it was mainly composed of mucinous material that was composed of glycoprotein and therefore amenable to the disintegration by bromelain and NAC.[10] On the other hand, the semi hard and hard mucin contained varying percentage of mucin that also disintegrated by the action of bromelain and NAC, the residual material that did not disintegrate were most probably not of glycoprotein in nature. The thiol content of the mucin does not suggest that they may play a role in mucin hardness since an equal percentage of S-H : S-S bonds were found in the three grades. The thiol (S-H and S-S) content may reflect on the amount of glycoprotein present in the three grades of mucin, soft mucin has the highest thiol content with semi soft having intermediate level. The dissolution experiments on the three mucin types have also indicated that soft mucin was solubilised completely, the semi hard with $60 \%$ solubilisation whilst the hard had about $30-40$ solubilisation. The remanent materials were of cellular origin. Hence, the thiol content of the three grades of mucin may in fact confirm the percentage of glycoprotein present in each one of them. The ratio of thiols being $3: 2: 1$ for soft, semi hard and hard mucin respectively that corresponds with the residual material left after dissolution, none in soft , 40\% in semi hard and $60-70$ $\%$ in the hard mucin. Since, the mucolytic disintegrated all the mucinous materials; it meant that soft mucin had a $100 \%$ mucinous material, whilst it was $60 \%$ in semi soft and about 30- $40 \%$ in hard mucin. Therefore the ratio of mucinous material in the three grades was approximately $3: 2: 1$ that seems to agree with thiol ratios.

All the three MUCs are of secretory types[29,30] and with regards to their contribution to the texture of mucin needs to be investigated in future studies. The relative proportion of MUCs in the mucin may have implication on the pathobiology of the disease since earlier studies have indicated that diffuse peritoneal adenomucinosis (DPAM) with better prognosis tend to produce a higher percentage soft mucin compared to peritoneal mucinous carcinoma (PMCA). Amongst the mucins examined in this study, MUC5B have been shown to be differentially glycosylated under the influence of estrogen during ovulation, enabling the thinning of mucinous barrier for better sperm penetration.[30,31] Hence, compactness of mucin may largely depend on glycosylation as well as inter molecular disulphide linkages present in the three mucins. Therefore the relative expression of these mucins may influence the texture and firmness (hardness) of the mucinous mass.[32,33]

The current mucolytic comprising of $300 \mu \mathrm{g} / \mathrm{ml}$ bromelain and $250 \mathrm{mM}$ N-acetyl cystein has capability of disintegrating mucin within 3 hours at $37 \mathrm{deg}$ Celsius, with in vivo evaluation suggesting that $48-76$ hours was required to acquire the same results.[10] However, based on the abundance of components such as lipids that ranges from $300-525 \mu \mathrm{g} / \mathrm{g}$ of mucin and sialic acid $(2.27-10.88) \mu \mathrm{M} / \mathrm{g}$ the addition of other suitable reagents to the current formulation, may further enhance the efficacy of our mucolytic. We may be able to reduce the time factor required for complete dissolution and at the same time may also affect further dissolution of both semi hard and hard mucin. Hence, further work in this area is required to improve the performance of our present mucolytic.

\section{Competing Interests}

The authors have declared that no competing interest exists.

\section{References}

1. Smeenk RM, van Velthuysen ML, Verwaal VJ, Zoetmulder FA. Appendiceal neoplasms and pseudomyxoma peritonei: a population based study. Eur J Surg Oncol 2008; 34: 196-201.

2. Bevan KE, Mohamed F. Moran BJ. Pseudomyxoma peritonei. World J Gastrointest Oncol 2010; 2: 44-50.

3. Gough DB, Donohue JH, Schutt AJ, Gonchoroff N, Goellner JR, Wilson TO, et al. Pseudomyxoma peritonei. Long-term patient survival with an aggressive regional approach. Ann Surg 1994; 219: 112-119.

4. Moran BJ, Cecil TD. The etiology, clinical presentation, and management of pseudomyxoma peritonei. Surg Oncol Clin N Am 2003; 12: 585-603.

5. Sugarbaker PH. Cytoreductive surgery and peri-operative intraperitoneal chemotherapy as a curative approach to pseudomyxoma peritonei syndrome. Eur J Surg Oncol 2001; 27: 239-243.

6. Chua TC, Moran BJ, Sugarbaker PH, Levine EA, Glehen O, Gilly FN, et al. Early- and long-term outcome data of patients with pseudomyxoma peritonei from appendiceal origin treated by a strategy of cytoreductive surgery and hyperthermic intraperitoneal chemotherapy. J Clin Oncol 2012; 30: 2449-2456.

7. Murphy EM, Sexton R, Moran BJ. Early results of surgery in 123 patients with pseudomyxoma peritonei from a perforated appendiceal neoplasm. Dis Colon Rectum 2007; 50: 37-42.

8. Smeenk RM, Verwaal VJ, Antonini N, Zoetmulder FA. Survival analysis of pseudomyxoma peritonei patients treated by cytoreductive surgery and hyperthermic intraperitoneal chemotherapy. Ann Surg 2007; 245: 104-109.

9. Miner TJ, Shia J, Jaques DP, Klimstra DS, Brennan MF, Coit DG. Long-term survival following treatment of pseudomyxoma peritonei: an analysis of surgical therapy. Ann Surg 2005; 241: 300-308.

10. Pillai K, Akhter J, Chua TC, Morris DL. A formulation for in situ lysis of mucin secreted in pseudomyxoma peritonei. Int J Cancer 2014; 134(2):478-86. 
11. Amini A, Ehteda A, Masoumi Moghaddam S, Akhter J, Pillai K, Morris DL. Cytotoxic effects of bromelain in human gastrointestinal carcinoma cell lines (MKN45, KATO-III, HT29-5F12, and HT29-5M21). Onco Targets Ther 2013; 6: 403-409.

12. Mall AS, Chirwa N, Govender D, Lotz Z, Tyler M, Rodrigues J, et al. MUC2, MUC5AC and MUC5B in the mucus of a patient with pseudomyxoma peritonei: biochemical and immunohistochemical study. Pathol Int 2007; 57: 537-547.

13. Carlstedt I, Lindgren $\mathrm{H}$, Sheehan JK, Ulmsten U, Wingerup L. Isolation and characterization of human cervical-mucus glycoproteins. Biochem J 1983; 211: 13-22.

14. Fries N, Dreyer M. An analytic solution of capillary rise restrained by gravity. J Colloid Interface Sci 2008; 320: 259-263.

15. Dan-Asabe B YS, Yawas DS , Aku SY. Water Displacement and Bulk DensityRelation Methods of Finding Density of Powdered materials. Int J of Innov Res in Sc, Eng and Tech 2013; 2:

16. Lowry OH, Rosebrough NJ, Farr AL, Randall RJ. Protein measurement with the Folin phenol reagent. J Biol Chem 1951; 193: 265-275.

17. Masuko T, Minami A, Iwasaki N, Majima T, Nishimura S, Lee YC. Carbohydrate analysis by a phenol-sulfuric acid method in microplate format. Anal Biochem 2005; 339: 69-72.

18. Schmid T, Panne U, Niessner R, Haisch C. Optical absorbance measurements of opaque liquids by pulsed laser photoacoustic spectroscopy. Anal Chem 2009; 81: 2403-2409.

19. Markely LR, Ong BT, Hoi KM, Teo G, Lu MY, Wang DI. A high-throughput method for quantification of glycoprotein sialylation. Anal Biochem 2010; 407: 128-133.

20. Balsamo R, Lanata L, Egan CG. Mucoactive drugs. Eur Respir Rev 2010; 19: 127-133.

21. Winger AM, Taylor NL, Heazlewood JL, Day DA, Millar AH. Identification of intra- and intermolecular disulphide bonding in the plant mitochondrial proteome by diagonal gel electrophoresis. Proteomics 2007; 7: 4158-4170.

22. Yusuf S, Nok AJ, Ameh DA, Adelaiye AB, Balogun EO. Correlation of gastric mucosal damage with sialic acid profile in rats: effect of hydrochloric acid, pepsin and hypertonic saline. Cell Biochem Funct 2005; 23: 339-345.

23. Miner-Williams W, Moughan PJ, Fuller MF. Methods for mucin analysis: a comparative study. J Agric Food Chem 2009; 57: 6029-6035.

24. Mantle M, Stewart G, Zayas G, King M. The disulfide-bond and rheological properties of intestinal mucins from normal subjects and patients with cystic fibrosis. Biochem J 1990; 266:597-604.

25. Mall AS, Tyler M, Lotz Z, Davidson A, Rodrigues J, van der Watt G,et al. The characterisation of mucin in a mature ovarian teratoma occuring in an eight year old patient. Int J Med Sci 2007; 4(2):115-123.

26. Mall AS, Lotz Z, Tyler M, Goldberg P. Immunohistochemical and Biochemical Characterisation of Mucin in Pseudomyxoma Peritonei: A case Study. Case Rep Gastroenterol 2011; 5:5-16.

27. Skrypek N, Duchene B, Hebbar M, Leteurtre, van Seuningen I, Jonckheere N. The MUC4 mucin mediates gemcetabine resistance in human pancreatic cancer cells via the Concenterative Nucleoside transporter family. Oncogene 2013; 32:1714-1723

28. Bafna S, Kaur S, Momi N, Batra SK. pancreatic cancer cells resistance to gemcetabine: the role of MUC4 mucin. Br J Can 2009; 101:1155-1161.

29. Kim CH, Oh Y, Han K, Seo HW, Kim D, Kang I et al. Expression of secreted mucins (MUC2, MUC5AC, MUC5B, and MUC6) and membrane-bound mucin (MUC4) in the lungs of pigs experimentally infected with Actinobacillus pleuropneumoniae. Res Vet Sci 2012; 92: 486-491.

30. Andersch-Bjorkman Y, Thomsson KA, Holmen Larsson JM, Ekerhovd E,Hansson GC. Large scale identification of proteins, mucins, and their O-glycosylation in the endocervical mucus during the menstrual cycle. Mol Cell Proteomics 2007; 6: 708-716.

31. Gipson IK, Moccia R, Spurr-Michaud S, Argueso P, Gargiulo AR, Hill JA, et al. The Amount of MUC5B mucin in cervical mucus peaks at midcycle. J Clin Endocrinol Metab 2001; 86: 594-600.

32. Cooper CA, Wilkins MR, Williams KL, Packer NH. BOLD--a biological O-linked glycan database. Electrophoresis 1999; 20: 3589-3598.

33. Hollingsworth MA, Swanson BJ. Mucins in cancer: protection and control of the cell surface. Nat Rev Cancer 2004; 4: 45-60. 